APS

physics

This is the accepted manuscript made available via CHORUS. The article has been published as:

\title{
Optical and electronic properties of dense sodium
} Miriam Marqués, Mario Santoro, Christophe L. Guillaume, Federico A. Gorelli, Julia Contreras-García, Ross T. Howie, Alexander F. Goncharov, and Eugene Gregoryanz Phys. Rev. B 83, 184106 - Published 17 May 2011 DOI: 10.1103/PhysRevB.83.184106 


\title{
On Optical and Electronic Properties of Dense Sodium
}

\author{
Miriam Marqués ${ }^{1}$, Mario Santoro ${ }^{2}$, Christophe L. Guillaume ${ }^{1}$, Federico Gorelli ${ }^{2}$, \\ Julia Contreras-García ${ }^{3}$, Ross Howie ${ }^{1}$, Alexander F. Goncharov ${ }^{4}$ and Eugene Gregoryanz ${ }^{1}$ \\ ${ }^{1}$ Centre for Science at Extreme Conditions and School of Physics and Astronomy, University of Edinburgh, Edinburgh, \\ ${ }^{2}$ European Laboratory for Non-Linear Spectroscopy, University of Florence, Florence, Italy \\ 3 Department of Chemistry, Duke University, Durham, NC, USA \\ ${ }^{4}$ Geophysical Laboratory, Carnegie Institution of Washington, Washington D.C., USA
}

\begin{abstract}
High-resolution Raman spectroscopy of the dense sodium reveals new low energy lattice excitations in the three high-pressure phases $c I 16, o P 8$ and $t I 19$ from 108 to $178 \mathrm{GPa}$. The Raman-active lattice modes indicate profound changes of the chemical bonding in $\mathrm{Na}$ upon its transformation to an insulating state ( $h P 4$ phase) at pressures above 178(2) GPa. The calculated values of the valence electron localization maxima versus pressure provide insights into the experimentally observed solidsolid phase transitions and anomalous melting behavior of sodium.
\end{abstract}

The profound changes in elements induced by the extreme conditions are fundamental to a broad range of problems in physics. The group-I alkali metals have been the testing grounds of the simple systems, which were predicted to adopt low symmetry structures upon compression $^{1,2}$. The proposed instability of cubic phases of $\mathrm{Li}$ under compression and the prediction that the low symmetry phases will take over at higher pressures ${ }^{2}$ were later confirmed experimentally both for $\mathrm{Li}$ and $\mathrm{Na}^{3-7}$. The appearance of open and incommensurate structures have been explained in terms of Peierls distortions ${ }^{2,8}$, $\mathrm{s} \rightarrow \mathrm{p}$ and $\mathrm{s} \rightarrow \mathrm{d}$ electronic transitions ${ }^{3}$, Fermi surfaceBrillouin zone interactions ${ }^{9,10}$ and more recently in terms of a combined effect of Couloumb repulsion, Pauli exclusion and orbital orthogonality that results in an increase of valence electrons in interstitial regions ${ }^{11}$. Theory also suggested that as density rises both $\mathrm{Li}$ and $\mathrm{Na}$ would become increasingly less metallic approaching a semiconducting phase; several candidates structures were proposed at very high pressures including $o C 8$ and $h P 4^{12,13}$. Indeed, the experimental studies ${ }^{5,14}$ reported the existence of Raman activity, color change and reflectivity decrease in Na signalling profound electronic modifications. The optical reflectivity measurements and electronic structure calculations demonstrated that Na transforms from a high-reflecting free-electron metal at ambient conditions to the nearly insulating $o P 8$ phase with significantly reduced reflectivity at $\mathrm{P}>118 \mathrm{GPa}$ and suggested that compression of the $o P 8$ phase beyond its range of stability would result in a metal-insulator transition at a 5 -fold reduction in volume ${ }^{15}$. The direct resistivity measurements indicated that $\mathrm{Li}$ exhibit a zero-gap semiconductor behavior at above $78 \mathrm{GPa}^{16}$ while optical spectroscopy and x-ray diffraction showed that at $\sim 200$ GPa Na turns into a wide gap insulator having the $h P 4$ structure $^{17}$. Besides predicted by theory high density low symmetry structures, some quite unexpected phenomena were observed in light alkalis such as pressure induced superconductivity ${ }^{18}$ and anomalously low melting temperatures at high compressions ${ }^{6,19}$. Even though the physical mechanisms responsible for the decreasing melting temperatures over a wide pressure range for $\mathrm{Na}$ and Li were extensively studied ${ }^{20-25}$ there is no general agreement and the phenomenon still requires a better understanding.

Although Raman spectroscopy of metals at high pressures is used as a probing tool ${ }^{26,27}$ is not widely applied due to several factors such as a very weak scattering and a strong background from the sample environment. The appearance of low symmetry structures in Na may give rise to Raman activity enabling to study vibrational dynamics and chemical bonding. In this paper we report Raman measurements on Na from 100 to $200 \mathrm{GPa}$ at a wide temperature range. In the lower pressure phases (cI16, oP8 and tI19) we observe new Raman peaks below $200 \mathrm{~cm}^{-1}$ while the highest pressure phase $(h P 4)$ has only one intense band at $\sim 350 \mathrm{~cm}^{-1}$ indicating profound changes in the electronic structure and therefore change of bonding. Using ab-initio calculations we show that the changes of the interstitial electron localisation patterns in the solid above $60 \mathrm{GPa}$ are responsible for the previously observed unusual melting behaviour and for the rich polymorphism of dense sodium. We have conducted experiments on three separate $\mathrm{Na}$ samples, two of which were used in the structural and infra-red measurements in Refs. 5,14,15 reaching maximum pressures of 130 to $200 \mathrm{GPa}$ and temperatures ranging from 140 to $550 \mathrm{~K}$. A number of pressure/heating/cooling cycles were made on each sample, enabling us to establish reproducible behaviour. For the Raman spectroscopy we have used a triple grating monochromator (trivista555, Acton-Roper), coupled to a CCD detector (Princeton Instruments), with the first two stages operating in subtractive mode. In order to ensure that the detected signals are indeed Raman scattering emanating from the sample we performed our measurements using the $647.1,752.5 \mathrm{~nm}$ line of $\mathrm{a} \mathrm{Kr}^{+}$ion laser and $514.5 \mathrm{~nm}$ of $\mathrm{Ar}^{+}$laser as the excitation sources and collected anti-Stokes components of the Raman signal. With the exception of the insulating $h P 4$ phase the synchrotron $\mathrm{x}$-ray diffraction was used to identify the structural modification of the sample. The pressure was measured from the Ta equation of state $^{28}$ and correlated with the optically measured stressed diamond edge ${ }^{29}$. For further 
information on sample preparation and loading see Refs. 4-6.

First-principles total energy calculations were performed within the density-functional theory (DFT) formalism with a plane-wave pseudo-potential approach, as implemented in the Vienna ab initio simulation package ${ }^{30}$. We used the Perdew-Burke-Ernzerhof generalised gradient (GGA) exchange-correlation functional ${ }^{31}$ and the projector augmented wave (PAW) all-electron description of the electron-ion-core interaction ${ }^{32}$. Due to the extremely large compressions involved, we treat the $2 s$, $2 p$ and $3 s$ electrons as valence. Brillouin zone integrals were approximated using the method of Monkhorst and $\mathrm{Pack}^{33}$ and the energies converged with respect to k-point density $(21 \times 21 \times 21,13 \times 17 \times 11$ and $16 \times 16 \times 16 k$-meshes for $c I 16, o P 8$ and $h P 4$ structures, respectively) and to the plane wave cutoff $(850 \mathrm{eV})$. Phonon frequencies were calculated within density-functional perturbation theory as implemented in the Quantum-ESPRESSO package ${ }^{34}$ The total energies required in the calculation of the phonons were obtained using the same Perdew-Burke-Ernzerhof parametrisation of the exchange correlation potential ${ }^{31}$. The ion-electron interaction was described by an ultrasoft (Vanderbilt ${ }^{35}$ ) pseudo-potential with $2 s$ and $2 p$ as valence states. Brillouin-zone integrations were performed using the same grids as in the VASP calculations. The topological analysis of the electron localisation function (ELF) was performed with the CRITIC code ${ }^{36}$. To this end, the VASP optimised structures were recalculated with the CRYSTAL98 $\operatorname{code}^{37}$ in order to obtain allelectron wave functions. Additionally, we have used the ELK code ${ }^{38}$.

Up to $100 \mathrm{GPa} N a$ exists in the Raman inactive bcc and fcc structures. Above $100 \mathrm{GPa}$ sodium adopts the $c I 16$ structure with 8 atoms in the primitive cell and 5 active Raman modes. Our calculated Raman frequencies for $c I 16$ range from $\sim 70$ to $\sim 490 \mathrm{~cm}^{-1}$ at 115(1) GPa. Fig. 1 shows the observed Raman spectrum (Stokes and anti-Stokes) of the $c I 16$ phase consisting of an unresolved doublet at $70 \mathrm{~cm}^{-1}$. We assign the lower frequency peak to the $A_{1}$ mode, which is due to the change associated with symmetric atomic stretching movements. The Raman activity changes when $\mathrm{Na}$ enters in the $o P 8$ phase at pressures above $118(1) \mathrm{GPa}^{5}$. At this point significant redistribution of the electronic charges takes place in the solid (see below) with new higher frequency $(\omega>100$ $\mathrm{cm}^{-1}$ ) modes appearing. Group theory analysis for the oP8 phase yields 12 Raman active modes out of which which we are able to observe 8 . Inset to Fig. 1 shows the Raman spectra of the $o P 8$ phase measured upon increasing temperature. At $127 \mathrm{GPa}$ the Raman signal disappears at about $350 \mathrm{~K}$, indicative of the melting and confirming the low melting temperature previously observed in x-ray diffraction experiments ${ }^{19}$. Above 127(1) $\mathrm{GPa} \mathrm{Na}$ transforms to the incommensurate $t I 19$ phase with a pronounced reduction of the reflectivity (see Fig. 2 and Refs. 14,15). The Raman activity in the $t I 19$ phase is determined by the host structure $(t I 16)$ which has 8 allowed Raman modes while the guest has one atom per unit cell and has no Raman activity. We have observed all 8 modes which consist of quite intense triplet, at below $200 \mathrm{~cm}^{-1}$, and 5 weaker peaks of higher frequency (see Fig. 2). The calculations performed on the approximate structures of $t I 18(20)$ show further increase of the pseudo-gap opening with respect to the $o P 8$ phase, with the valence charge density accumulating along the guest chains $^{15}$. At pressures of $178(3) \mathrm{GPa}$ the $t I 19$ phase abruptly changes to a completely transparent insulating phase with very intense Raman signal (Fig. 2). The evolution of the optimized structural parameters of the $o P 8$ phase with pressure indicate that this phase would transform to the hexagonal structure $(h P 4)$ at around $240 \mathrm{GPa}$ in good agreement with Refs. 15,17.

We have calculated the electron localization function (ELF) for the $c I 16, o P 8$ and $h P 4$ phases of $\mathrm{Na}$ (see Fig. 3 and 4 for the ELF isosurfaces and contour plots, respectively). The topological analysis of ELF can reveal the bonding and localization pattern from first principles. Two stationary points are analyzed: attractors (ELF maxima) and bips ( $1^{\text {st }}$ order saddle points). In general, the ELF value approaches 1 in regions of space where electron pairing occurs (e.g. atomic shells, bonds and lone pairs), and 0 in the limit between those surfaces, where there is a high probability of finding electrons from different pairs. The value 0.5 corresponds to the homogeneous electron gas (HEG). Hence, the ELF profile in the valence region of simple metals is nearly flat and usually close to $0.5^{39}$ as would be expected for the bcc phase of $\mathrm{Na}$. Indeed, our calculations in the bcc phase at ambient pressure show a very homogenous electron distribution consisting of valence ELF attractors on tetrahedral $12 d$ sites with ELF value around 0.6 and joined through bips with similar ELF value. This profile is maintained until the bcc-fcc phase transition at $65 \mathrm{GPa}$. The fcc phase presents two different ELF attractors on octahedral and tetrahedral sites. Their corresponding ELF values are, respectively, higher and lower than the HEG value of 0.5 and monotonically increase/decrease with pressure. This is in agreement with the pressure-induced electron density increase on octahedral interstices at the expense of the tetrahedral sites proposed in Ref. 11. This trend is maintained in the $c I 16$ phase, with two differentiated ELF attractor regions of high and low ELF value around the tetrahedral $12 a$ and $12 b$ sites, respectively. Interestingly, the $c I 16$ phase is a distorted $2 \times 2 \times 2$ superstructure of the bcc structure and the ideal bcc structure at the experimental pressure range of $c I 16$ would have identical ELF attractors on tetrahedral sites with low ELF value of 0.38. As compression into oP8 takes place, the polarisability screening of the free electron present at lower pressures does not hold (compare $c I 16$ and $o P 8$ in Fig. 3 ) and intense modes appear in the Raman spectrum (Fig. 1). In the $o P 8$ phase only one attractor type on $4 c$ sites is present having values close to the unity. Although the localisation of interstitial electrons in $o P 8$ is 
deprived of the most characteristic HEG features, some of the metallic properties are still preserved. The localisation of electrons leads to the widening of the pseudo-gap but the exchange between valence electrons still takes place, as can be seen from the connection between different valence regions (see Fig. 4$)^{40}$. Moreover, the value of ELF at the bips connecting the different localised regions becomes smaller with increasing pressure eventually leading to an insulating structure. The small range of stability of this phase $(\sim 118$ to $\sim 125 \mathrm{GPa})$ and its transformation to the incommensurate $t I 19$ phase precludes this possibility (see also Ref. 15), shifting the metal-to-insulator transformation to much higher pressures. In the insulating phase, $h P 4$, (see Fig. 3(c)), the valence electrons localise even more strongly in the interstitial voids forming well-defined entities with very low ELF values at the bips connecting them highlighting their orthogonality. Curiously, a semiconducting $h P 4$ phase has also been reported for $\mathrm{K}$ transforming to the oP8 phase with pressure ${ }^{41}$. Moreover, these electrons can also be characterised as real electron pairs as can be seen from the high ELF value at the center of the interstices (0.9) (see Figs. 3 and 4). The band gap is fully open in this phase and reaches values of $\mathrm{E}_{g}=2.1 \mathrm{eV}$ as measured by the optical transmission spectroscopy (Fig. 2 , left panel). Interestingly, if we consider the attractors as pseudoanions without core ${ }^{41}$, the most intense Raman activity modes can be associated with the movements of the sodium atoms with respect to the valence attractors (see Fig. 5). The experimentally observed Raman mode has $\mathrm{E}_{2 g}$ symmetry. Its intensity is clearly influenced by the change caused by the atomic movements towards the maxima of the newly formed electron pairs (see Fig. 5). As such, this observation provides the first documented experimental proof of the pseudoanionic ELF attractors. We also tried to measure the Na melting temperatures above $130 \mathrm{GPa}$ in this study. However, heating of Na$t I 19$ at $140 \mathrm{GPa}$ up to $550 \mathrm{~K}$ has not melted the sample. One can try to estimate the melting temperatures from the Raman spectroscopy. We measured the temperature dependence of the $\mathrm{E}_{2 g}$ mode of the $h P 4$ phase at 180 GPa and $200 \mathrm{GPa}$ (see Fig. 6). This mode correlates with a transverse acoustic phonon; thus, its frequency shift provides information on the $\mathrm{C}_{44}$ elastic constant which represents the slope of this branch at the center of the Brillouin zone. The relation can be derived with a three-body force model for an hexagonal solid with a non-ideal c/a ratio ${ }^{42}$. In order to estimate $\mathrm{C}_{44}$ we have used the following formula ${ }^{42}$ :

$$
C_{44}=2 \pi^{2} M\left(\frac{\sqrt{3} c}{6 a^{2}}\right) \nu^{2}
$$

where $\mathrm{M}$ is the atomic mass, a,c and $\nu$ are the measured lattice constants and Raman frequency respectively. The values of $\mathrm{C}_{44}$ in 180 to $200 \mathrm{GPa}$ pressure range as estimated from the frequencies of the $\mathrm{E}_{2 g}$ mode (120 GPa) and from first principles calculations (129 GPa) are in very good agreement. The main shear modulus of the hexagonal media is $\mathrm{G}=2 \mathrm{C}_{44}+\mathrm{C}_{66}$. The calculations for $\mathrm{C}_{66}$ give value of about $40 \mathrm{GPa}$ showing that the shear modulus of the Na-hP4 is mostly driven by $\mathrm{C}_{44}$. The measured temperature dependence of the $\mathrm{E}_{2 g}$ mode between 180 and $200 \mathrm{GPa}$ (see Fig. 6) is $\sim 0.1 \mathrm{~cm}^{-1} / \mathrm{K}$. This estimate suggests quite high temperatures (upper bound of $\mathrm{T}>3500 \mathrm{~K}$ ) needed to substantially decrease $\mathrm{G}$ and to melt Na- $h P 4$.

In Fig. 7 we show the ELF attractor values as function of pressure, together with the phase diagram of $\mathrm{Na}$. Remarkably, there is a clear correlation between pressure evolution of ELF attractors and solid-solid phase transitions, which suggests that the changes in the interstitial electrons distribution are indeed the driving force for these phase transitions. Also, pressure dependence of ELF attractors tightly correlates with the melting line. At the same pressures $(\mathrm{P}>30 \mathrm{GPa})$ when the melting temperatures passed their maximum and start to decrease, the ELF attractor value is slowly decreasing. When $\mathrm{Na}$ enters the pressure domain of the fcc phase, there is a splitting in two attractors with high and low ELF as described above. The ELF value at the lower ELF attractor monotonically decreases upon increasing pressure. This trend is maintained in the $c I 16$ phase and up to the $c I 16-o P 8$ phase transition point $(\sim 118$ GPa), which correlates with the negative slope in the melting line that reaches a minimum in the $c I 16$ phase. At the $c I 16-o P 8$ phase transition the ELF attractor value abruptely changes reaching typical high values of ionic bonding and, correspondingly, the melting line starts to steeply increase with pressure. The ELF attractor value is an efficient route to parametrize valence electron localization, which in turn is the source of binding energy and of all effects connected to binding energy, such as phase stabilization, bulk moduli, shear moduli and, ultimately, melting temperatures. The existence in the fcc and $c I 16$ phases of attractors with very low ELF value, which also decreases upon increasing pressure, is likely to indicate weaker binding. And, as a consequence of less bonded atoms a lower temperature is required to melt the solid. At above $118 \mathrm{GPa}(o P 8)$, the low ELF attractors dissapear, and the high ELF value of the only attractor indicates a change to a strong pseudoionic bonding which leads to high melting temperatures. Our estimates of the melting temperatures combined with the experimental points indicate temperatures in excess of $3500 \mathrm{~K}$ needed to melt sodium at around $200 \mathrm{GPa}$. The extrapolation of the melting curve above $30 \mathrm{GPa}$ using empirical laws which do not have negative component of the melting curve ${ }^{43}$ suggests (see Fig. 7) that the melting curve of $\mathrm{Na}$ not only would completely recover the high temperatures after passing through the deep minimum at 118 $\mathrm{GPa}$ but might reach much higher values than the simple extrapolation suggests.

We acknowledge the support from the U.K. Engineering and Physical Sciences Research Council; the EU Contract No. FP7 G.A. No 228334 laserlabeurope, and the Ente Cassa di Risparmio di Firenze supporting research 
at LENS under the grant "Firenze Hydrolab"; financial support from the Spanish MALTA Consolider Project; and support by DOE/NNSA (CDAC), Army Research Office and NSF-EAR-1015239.
1 A. McMahan, Phys. Rev. B 29, 5982 (1984).

2 J. Neaton, N. Ashcroft, Nature 400, 141 (1999).

${ }^{3}$ M. Hanfland et al., Nature 408, 174 (2000).

${ }^{4}$ M. McMahon et al., PNAS, 104, 17297 (2007).

${ }^{5}$ E. Gregoryanz et al., Science 320, 1054 (2008).

${ }^{6}$ C. Guillaume et al., Nature Physics, 7, 211 (2011)

7 M. Marqués et al., Phys. Rev. Lett., 106, 095502 (2011)

8 Zhou et al., J. Phys. Condens. Matter 21, 025508 (2009).

${ }^{9}$ G. Ackland, I. Macleod New J. Phys., 6, 138 (2004).

10 V. Degtyareva, Phys. Usp., 49, 369 (2006).

11 B. Rousseau and N. W. Ashcroft, Phys. Rev. Lett. 101, 046407 (2008).

12 J. Neaton, N. Ashcroft, Phys. Rev. Lett., 86, 2830 (2001).

13 N. Christensen, D. Novikov, S. State Comm. 119, 477 (2001).

${ }^{14}$ L. Lundegaard et al., Phys. Rev. B, 79, 064105 (2009).

15 A. Lazicki et al., PNAS, 106, 6525 (2009).

16 K. Shimizu and T. Matsuoka Nature 458, 186 (2009).

17 Y. Ma et al., Nature 458, 182 (2009).

18 K. Shimizu et al., Nature 419, 597 (2002); V. Struzhkin et al., Science 298, 1213 (2002);

19 E. Gregoryanz et al., Phys. Rev. Lett. 94, 185502 (2005).

20 E. R. Hernández and J. Íñiguez, Phys. Rev. Lett. 98, 055501 (2007).

21 J. Raty et al., Nature, 449, 448 (2007).

22 L. Koĉi et al., Phys. Rev. B 77, 132101 (2008).

23 M. Martinez-Canales and A. Bergara, J. of Phys. and Chem. of Solids, 692151 (2008)

${ }^{24}$ I. Tamblyn et al., Phys. Rev. Lett. 101, 075703 (2008).

25 E. Hernández et al., Phys. Rev. Lett. 104, 185701 (2010).
${ }^{26}$ H. Olijnyk, Phys. Rev. Lett., 68, 2232 (1992).

27 A. F. Goncharov and V.V Struzhkin, J. of Raman Spectroscopy, 34, 532 (2003).

28 M. Hanfland, K. Syassen and J. Kahler, J. Appl. Phys. 91, 4143 (2002).

29 Y. Akahama, and H. Kawamura, J. Appl. Phys. 96, 3748 (2004).

30 G. Kresse and J. Furthmuller, Phys. Rev. B. 54, 11169 (1996).

31 J. P. Perdew, K. Burke, and M. Ernzerhof, Phys. Rev. Lett. 77, 3865 (1996).

${ }^{32}$ G. Kresse and D. Joubert, Phys. Rev. B 59, 1758 (1999).

${ }^{33}$ H.J. Monkhorst and J. D. Pack, Phys. Rev. B 13, 5188 (1976).

34 The QUANTUM-ESPRESSO code is available at http://www.quantum-espresso.org.

35 D. Vanderbilt, Phys. Rev. B 41, 7892 (1990).

36 J. Contreras-García et al., J. Chem. Theory Comput. 5 (1), 164 (2009).

37 V. R. Saunders et al., CRYSTAL98 User's Manual (University of Torino, Torino, 1998.

38 http://elk.sourceforge.net

39 B. Silvi, and C. Gatti J. Phys. Chem. A, 104, 947 (2000).

40 J. Contreras-García and J. M. Recio, Theor. Chem. Acc. DOI 10.1007/s00214-010-0828-1

41 M. Marqués et al., Phys. Rev. Lett. 103, 115501 (2009).

42 J. C. Upadhyaya et al., Can. J. Phys. 72, 61 (1994).

43 E. Gregoryanz et al., Phys. Rev. Lett. 90, 175701 (2003). 


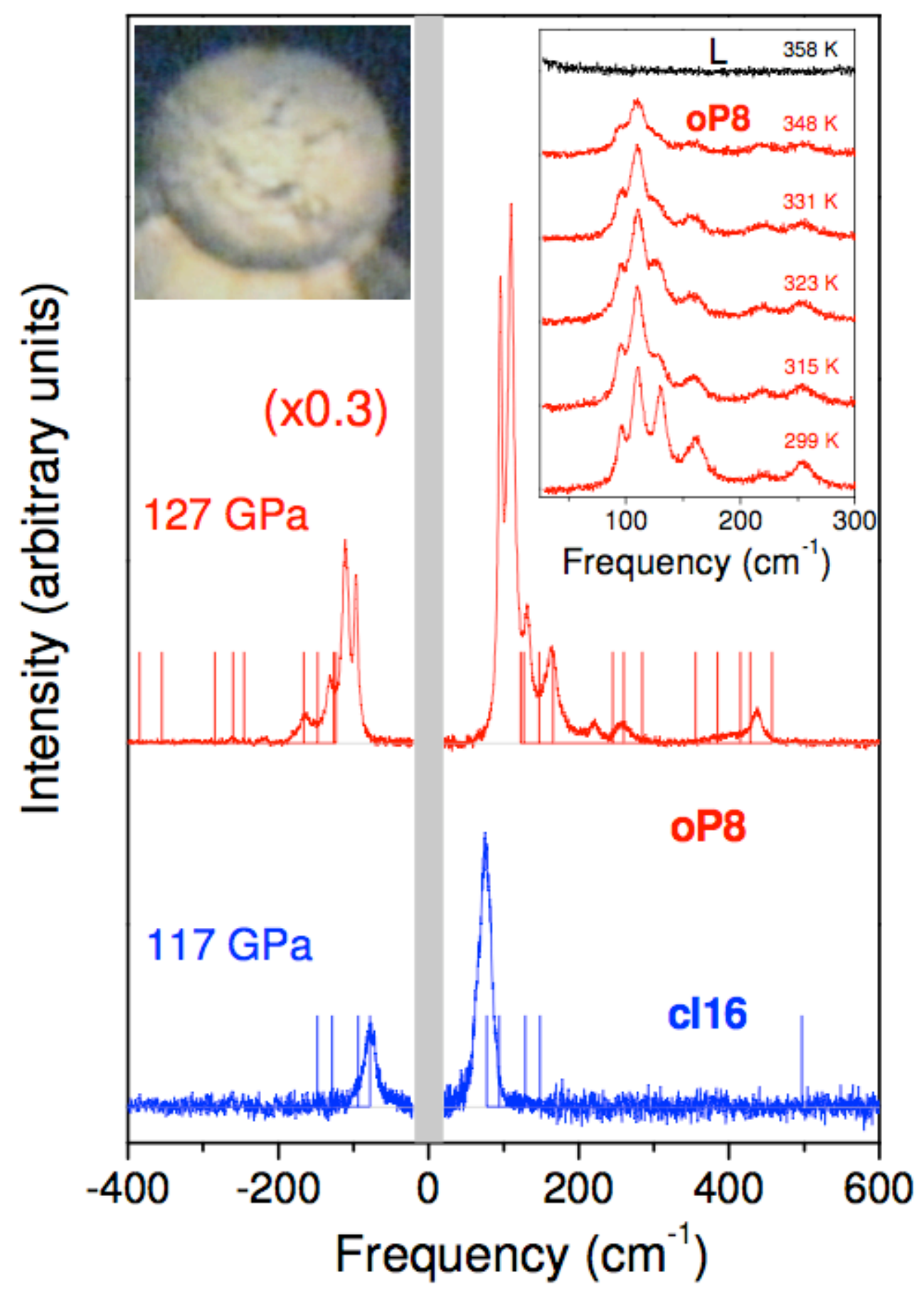

FIG. 1: (Color online) Representative Raman spectra of the $c I 16$ and $o P 8$ phases. The vertical bars indicate the calculated frequencies. Inset (right) : Raman spectra of the $o P 8$ phase at $127 \mathrm{GPa}$ taken at different temperatures. The disappearance of the spectra corresponds to the melting at around the minima of the melting curve. Smooth polynomial lines have been fitted to the broad luminescence background emanating from diamonds and to the wings of the Rayleigh line, and then subtracted to the spectra in both panels. The spectra have been vertically scaled for clarity. Inset (left): microphotograph of the sample in the $o P 8$ phase. 

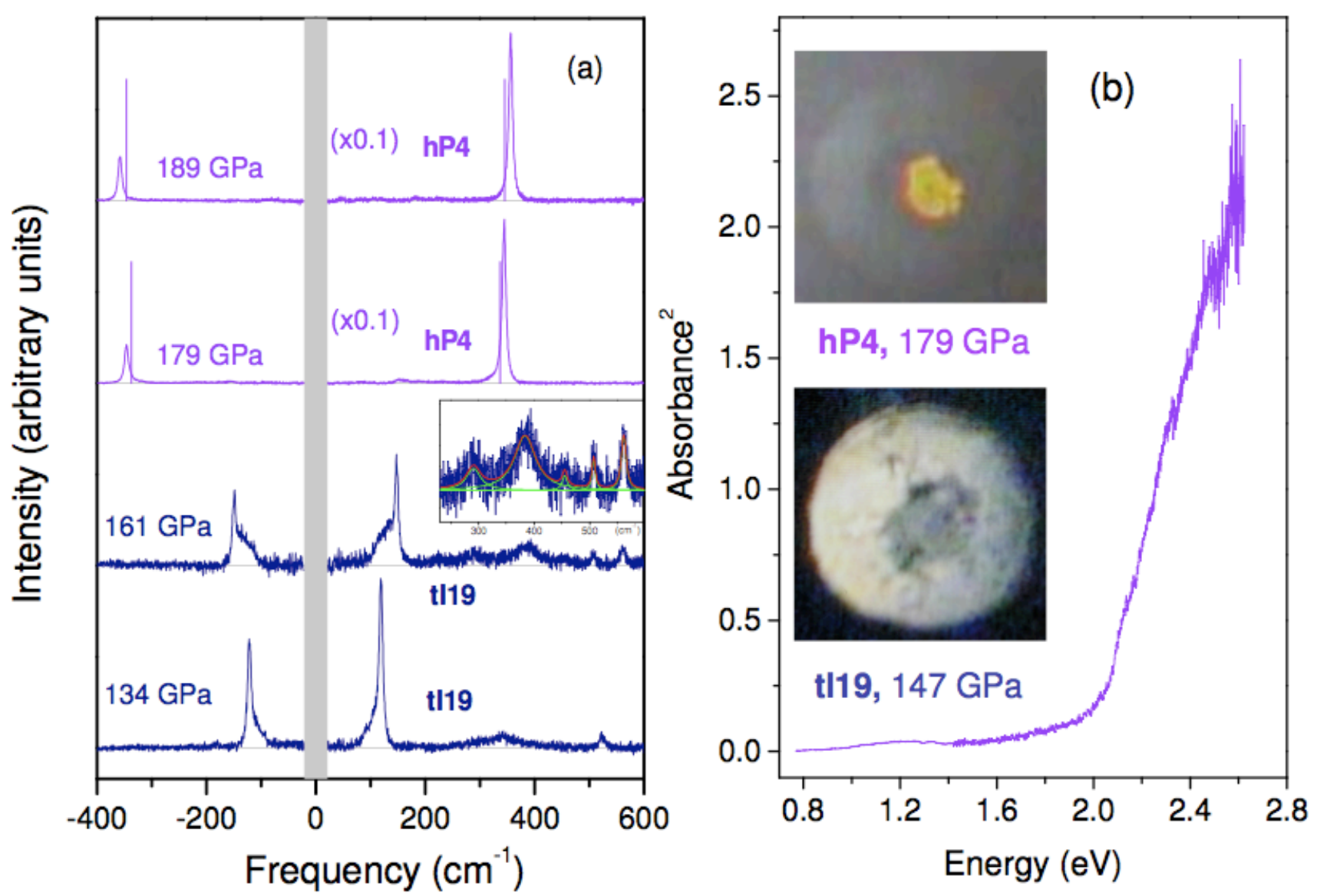

FIG. 2: (Color online) Left panel: representative Raman spectra of the $t I 19$ and $h P 4$ phases. The vertical bars indicate the calculated frequencies for the $h P 4$ phase. Inset: magnified high energy weak peaks in the $t I 19$ phase. Right panel: sample micro-photograph (top inset) and its optical absorption spectrum (absorbance to the second power) in the $h P 4$ phase. Bottom inset: Sample micro-photograph in the $t I 19$ phase. The photographs were taken under the transmitted and reflected light and show the same sample as in Fig. 1. 


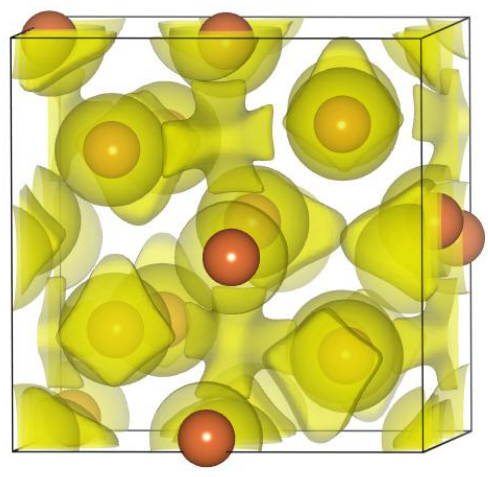

cl16

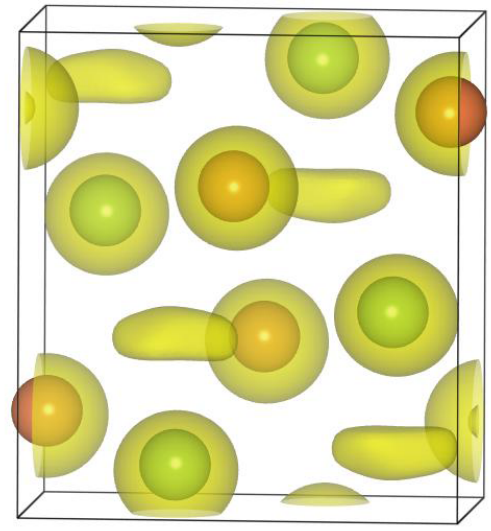

oP8

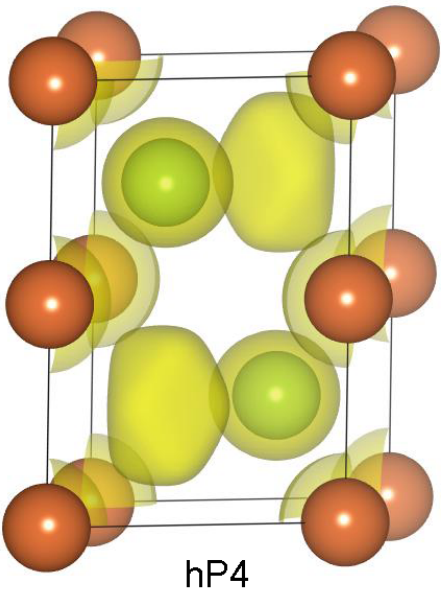

FIG. 3: (Color online) ELF isosurfaces (yellow online) for the $c I 16, o P 8$ and $h P 4$ structures. The ELF values are fixed at $0.25,0.5$ and 0.7 , respectively. The atomic positions are shown by spheres of different tonalities (online: red, green) representing non-equivalent atomic sites. The red spheres correspond to the equivalent $\mathrm{Na}$ atoms of the $c I 16$ structure, one $4 c$ site of the $o P 8$ structure and the $2 a$ site of the $h P 4$ structure. The green spheres correspond to the other $4 c$ site of the $o P 8$ structure and the $2 c$ site of the $h P 4$ structure.

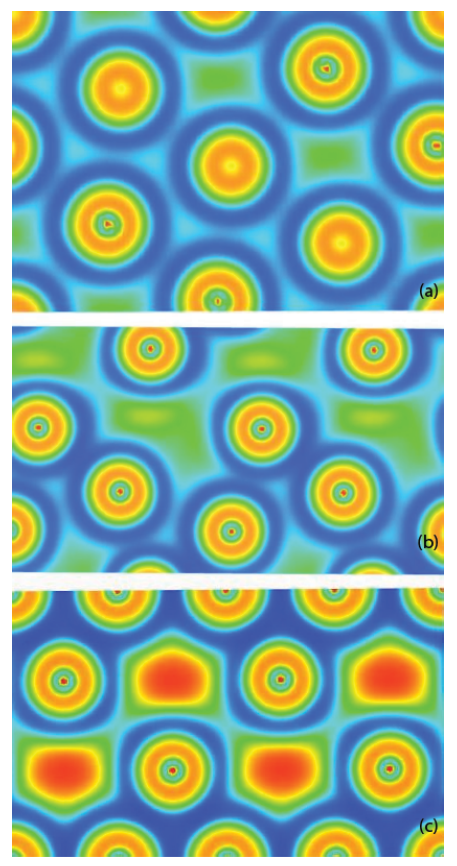

FIG. 4: (Color online) ELF contour plots for the (a) (110) plane of $c I 16$ (b) (040) plane of $o P 8$ and the (c) (110) plane of $h P 4$, respectively. The ELF values range from 0 (blue online) to 1 (red online). 


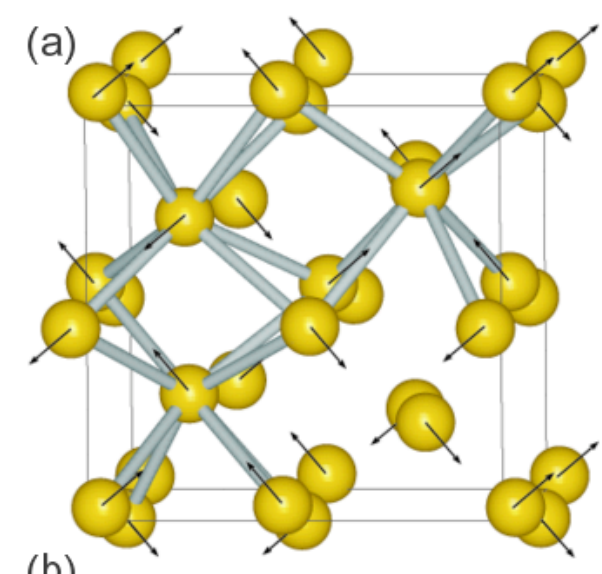

(b)

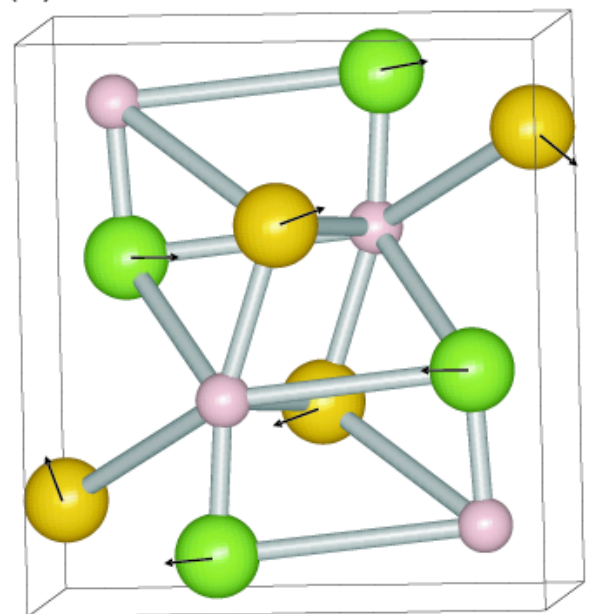

(c)

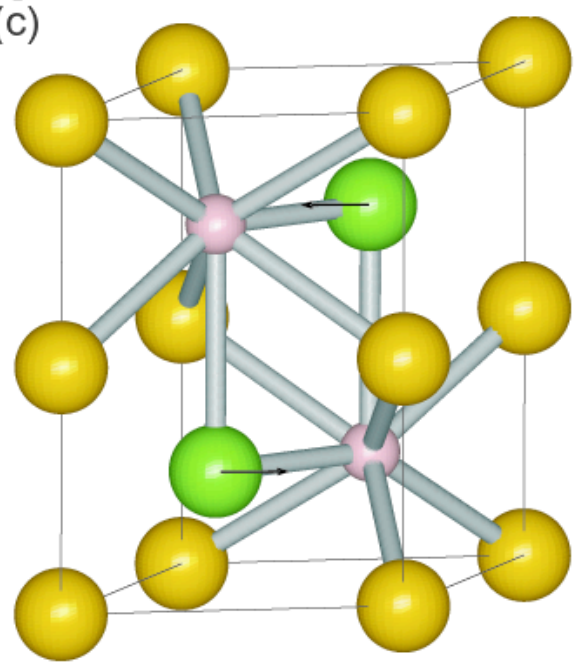

FIG. 5: (Color online) Atomic movements showing observed Raman modes in $c I 16, o P 8$ and $h P 4$ phases. (a) $c I 16-\mathrm{A}_{1}$ mode (b) $o P 8-\mathrm{A}_{g}$ mode (c) $h P 4-\mathrm{E}_{2 g}$ mode. The yellow spheres correspond to the equivalent $\mathrm{Na}$ atoms of the $c I 16$ structure, one $4 c$ site of the $o P 8$ structure and the $2 a$ site of the $h P 4$ structure. The green spheres correspond to the other $4 c$ site of the $o P 8$ structure and the $2 c$ site of the $h P 4$ structure. The smaller spheres (pink online) represent the ELF valence attractor maxima. 

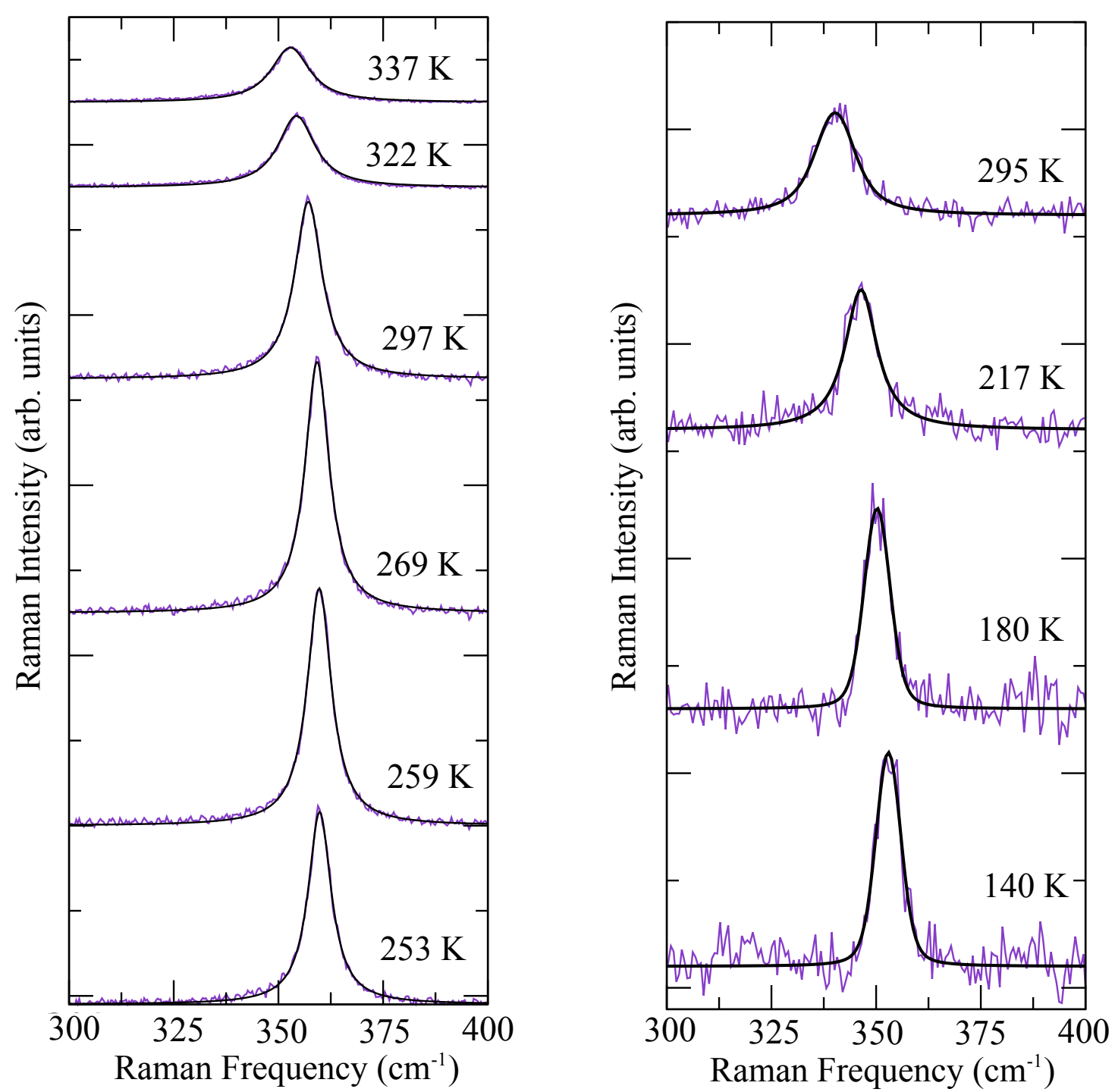

FIG. 6: Representative Raman spectra of the $h P 4$ phases at $200 \mathrm{GPa}$ (left panel) and $180 \mathrm{GPa}$ (right panel) at different temperatures. The spectra have been vertically scaled for clarity. 


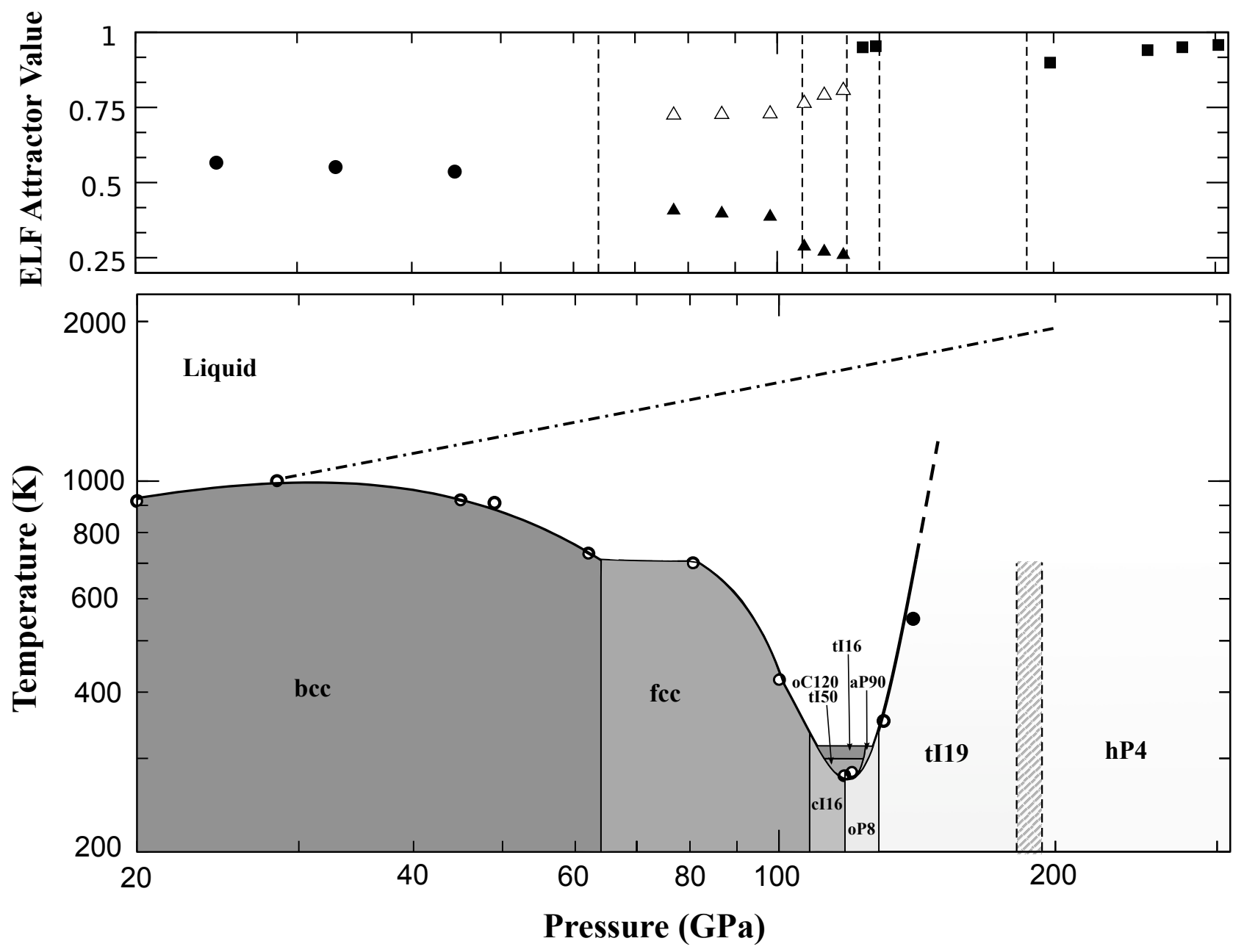

FIG. 7: Lower panel: Proposed phase diagram of Na. The lower pressure region, shown in open circles, is adapted from the Refs. 5,19; the solid circle is measurement in this study; the dot-dashed line shows the extrapolation of the melting curve from $30 \mathrm{GPa}$ with the Simon-Glatzel empirical law ${ }^{43}$; the dashed line extrapolating the melting curve above 140 $\mathrm{GPa}$ is a guide to eye only, the vertical dashed lines show the region of the $t I 19$ and $h P 4$ co-existence. Upper panel: ELF attractor values as function of pressure in different solid phases. The solid circles show the ELF attractor values in the bcc phase, empty and solid triangles show the ELF attractor values at non-equivalent interstitial sites (octahedral and tetrahedral sites, respectively, in fcc and tetrahedral $12 a$ and $12 b$ sites, respectively, in cI16). Solid squares show the ELF attractor values in the $o P 8$ and $h P 4$ phases. 